\section{Genetics of cardiomyopathies in children}

\author{
Matteo Vatta, Jeffrey A. Towbin ${ }^{2}$ \\ 1 Department of Molecular and Human \\ Genetics, Baylor College of Medicine, \\ Houston, TX; ${ }^{2}$ The Heart Institute, \\ Cincinnati Children's Hospital Medical \\ Center, Cincinnati, OH, USA
}

\section{Abstract}

Cardiomyopathies are diseases of the heart muscle leading to heart failure and/or an increased risk of arrhythmogenic sudden cardiac death. These disorders represent a major cause of morbidity and mortality in children. In childhood forms of cardiomyopathy, genetic etiologies are frequent, but non-genetic or acquired causes, such viral infection, also play a significant role. In the last twenty years, the genetic causes of cardiomyopathies have been increasingly identified and clinical correlations are beginning to be defined. Here we present an overview of the recent advances in our understanding of the genetics of cardiomyopathies in children and what is known about the pathophysiological mechanisms underlying these gene-related forms of disease.

\section{Introduction}

Primary cardiomyopathies remain a major cause of morbidity and mortality in children with an estimated incidence of 1.13/100,000 cases in the U.S., and they represent the leading cause of transplantation in children over one year of age. ${ }^{1,2}$ Several classified forms of cardiomyopathy exist which have diverse clinical, structural, morphological, and functional presentations. These forms include dilated cardiomyopathy (DCM), hypertrophic cardiomyopathy (HCM), left ventricular non-compaction (LVNC), arrhythmogenic right ventricular cardiomyopathy (ARVC) and restrictive cardiomyopathy (RCM). ${ }^{1-4}$ Among the various types of cardiomyopathies, DCM represents approximately $40 \%$ of all cases in children, followed by HCM, LVNC and more rarely by ARVC and RCM.1-4After over two decades of genetic and molecular research, many causative genes have been identified and overlap in the genetic causes of the various forms of cardiomyopathies have also been identified, in which defects in the same gene could lead to allelic disorders. ${ }^{1-4}$ Here, we will briefly describe the most important forms of cardiomyopathy and what is currently known about their genetic basis and the pathophysiological mechanisms responsible for the disorders.

\section{Dilated cardiomyopathy}

DCM is characterized by an enlarged left ventricular chamber, left ventricular wall thinning and systolic dysfunction. ${ }^{1-4}$ Individuals with DCM commonly present with symptomatic heart failure, arrhythmias or conduction disturbance. ${ }^{1-4}$ DCM in children has an estimated incidence of approximately $0.57 / 100,000$ cases compared to the report of $1 / 2,500$ incidence in adult subjects. ${ }^{2}$ However, the actual incidence could be far higher due to reduced detection rate caused by incomplete penetrance and variable expressivity observed in DCM, which can lead to a prolonged asymptomatic period preceding the development of overt heart failure.

Although a significant proportion of childhood DCM could be attributed to infections causing myocarditis (16\%), ${ }^{5,6}$ a large fraction of pediatric DCM can be genetic in origin, either sporadic, if the child has no previous family history and screening of first degree relatives is negative, or familial if it occurs in two or more close relatives. ${ }^{1-7}$ Currently, familial DCM is estimated to occur in up to $67 \%$ of cases after accurate screening of the relatives of idiopathic DCM subjects. ${ }^{8}$

A significant percentage of DCM cases may be explained by mutations in single genes that affect critical pathways of contractile function, ion distribution, or cellular function. This was initially demonstrated by the breakthrough identification of dystrophin, the gene responsible for Duchenne (DMD) and Becker muscular dystrophy (BMD) and the associated skeletal myopathy and DCM, as the gene responsible for the X-linked form of DCM (XLCM) by Towbin and colleagues in $1993 .{ }^{9}$ However, defects in the $D M D$ gene could explain only a fraction of all DCM cases; later, other genes responsible for DCM were identified, defining DCM as a genetically heterogeneous entity. Based on these findings, we formulated the final common pathway hypothesis (1998) in which it was suggested that abnormalities in other genes encoding for dystrophin-associated proteins and proteins involved in the structural formation and maintenance of cardiomyocyte structure and contractile function could also potentially lead to the development of DCM. ${ }^{10}$ It is now known that perturbation of cardiomyocyte proteins involved in contractile force generation and transmission, such as cytoskeletal, sarcomeric, ion channel and transcription factor proteins, are involved in the pathogenesis of DCM. ${ }^{7}$ In vitro models and animal models recapitulating the human disease suggest that alteration of the protein continuum connecting the cardiomyocyte plasma membrane (sarcolemma) to the sarcomere, and through the intermediate filaments, to the
Correspondence: Matteo Vatta, Department of Molecular and Human Genetics, Baylor College of Medicine, Houston, TX, USA.

E-mail:mvatta@bcm.edu

Key words: sudden cardiac death, dilated cardiomyopathy, hypertrophic cardiomyopathy, left ventricular noncompaction, restrictive cardiomyopathy, arrhythmogenic right ventricular cardiomyopathy, Nav1.5, LQTS, ion channels.

Received for publication: 16 May 2011.

Accepted for publication: 20 July 2011.

This work is licensed under a Creative Commons Attribution NonCommercial 3.0 License (CC BYNC 3.0).

(C) Copyright M. Vatta and J.A. Towbin, 2011

Licensee PAGEPress, Italy

Cardiogenetics 2011; 1:e9

doi:10.4081/cardiogenetics.2011.e9

perinuclear membrane, can lead to a transient hypertrophic phase, followed by decompensated systolic performance, left ventricular wall thinning and left ventricular chamber dilation. ${ }^{7}$

Currently, approximately 33 genes have been identified to cause DCM in isolation, demonstrating a high level of genetic heterogeneity (Table 1), and many private mutations have been observed in unrelated subjects suggesting a high level of allelic heterogeneity. ${ }^{7}$ In addition, several genes have also been identified that are associated with syndromic forms of DCM, such as genes involved in metabolism and mitochondrial function, besides loci allelic to other cardiomyopathic phenotypes. ${ }^{7,11}$ In particular, primary diseases of the skeletal muscle, such as various forms of muscular dystrophy and other skeletal myopathies frequently present with a DCM phenotype..$^{12,13}$

Despite the large number of genes associated with DCM, they account for only approximately $40 \%$ of all cases, with LMNA (5\%), MYH7 (4\%), and TNNT2 (3\%) being the most frequently involved in familial DCM, while mutations in all associated sarcomeric genes cover only up to $10 \%$ of all DCM cases. ${ }^{7}$

In addition, genes encoding for ion channels such as the cardiac sodium channel (SCN5A), which causes long-QT syndrome (LQTS) and Brugada syndrome, as well as $A B B C 9$, coding for the Kir6.2 regulatory subunit, an inwardly rectifying cardiac KATP channel, are implicated in the pathogenesis of DCM. ${ }^{7}$ This suggests that ion channels not only influence the electrocardiographic (ECG) findings and potentiate arrhythmias in DCM, ${ }^{14}$ but may also weaken the cardiomyocytes structure leading to DCM and vice versa, as recently reported in a mouse model harboring the a $L D B 3$-encoded ZASP mutation causing DCM in humans. ${ }^{15}$ 
Another potential mechanism, particularly in the case of the SCN5A-ecnoded Nav1.5, is its relationship with dystrophin. $\mathrm{Na}_{\mathrm{v}} 1.5$ binds to dystrophin and could potentially disrupt the function of dystrophin and lead to a dystrophin-related cardiomyopathy, as would be predicted by the final common pathway hypothesis. Clinically, the presentation of idiopathic, acquired and genetic forms of DCM is indistinguishable. This suggests that clinical evaluation of relatives of children with DCM should always be considered when a diagnosis of DCM is reached. Unfortunately, a significant proportion of DCM remains idiopathic, where a firm etiologic diagnosis cannot be reached. This suggests that many more genes are yet to be discovered, and that genetic testing in the clinical setting using the standard Sanger sequencing technique appears to have limitations due to the large number of genes involved. It is likely that the molecular diagnosis of DCM will benefit from the clinical application of high throughput technologies such as whole genome or exome sequencing, multiplex ligation-dependent probe amplification (MLPA), array comparative genomic hybridization (aCGH) and high density arrays combining single nucleotide polymorpshism (SNP) and copy number variation (CNV) probes using the currently available platforms. ${ }^{16,17}$

\section{Hypertrophic cardiomyopathy}

Hypertrophic cardiomyopathy is one of the most common genetic disorders with a prevalence of $1 / 500$, representing also the most frequent cause of sudden cardiac death in young athletes in the United States..$^{3-4} \mathrm{HCM}$ is characterized by excessive thickening generally limited to the left ventricular myocardium and interventricular septum, in the absence of insults that increase after load such as aortic stenosis or hypertension, and morphologically is typically characterized by myocyte disarray. ${ }^{3-}$ ${ }^{4}$ The interventricular septal thickening most commonly demonstrates asymmetric hypertrophy but focal areas of septal hypertrophy or concentric hypertrophy may occur. ${ }^{3-4}$ Left ventricular outflow tract obstruction may also occur. Individuals with HCM may be asymptomatic or present signs of sudden death or heart failure. Clinical presentations therefore include syncope or non-resuscitated sudden death, dyspnea, diaphoresis, chest pain, palpitations, or arrhythmias. The age of onset of HCM-related symptoms varies from infancy to adulthood. However, most appear in adolescence. As previously described for DCM, primary HCM has a genetic basis in most cases. Approximately 21 genes, mostly encoding for sarcomeric proteins and mainly involved in force generation, have been identified to cause HCM, and many of them are allelic to DCM (Table 1). ${ }^{18}$

Similar to DCM, HCM is characterized by
Table 1. Genes associated with cardiomyopathies and their allelic disorders.

\begin{tabular}{|c|c|c|c|c|}
\hline Gene & OMIM* & Locus & Gene name $^{\circ}$ & $\begin{array}{l}\text { Associated } \\
\text { phenotypes }\end{array}$ \\
\hline$A B C C 9$ & 601439 & 12p12.1 & ATP-binding cassette, sub-family C, member 9 & DCM \\
\hline ACTCl & 102540 & $15 q 14$ & Actin, alpha, cardiac muscle 1 & $\begin{array}{l}\text { DCM } \\
\text { HCM } \\
\text { RCM }\end{array}$ \\
\hline ACTN2 & 102573 & $1 q 42-q 43$ & Actinin, alpha 2 & $\begin{array}{l}\text { DCM } \\
\text { HCM }\end{array}$ \\
\hline$B A G 3$ & 603883 & $10 q 25.2-q 26.2$ & BCL2-associated athanogene 3 & $\begin{array}{l}\text { MFM } \\
\text { DCM }\end{array}$ \\
\hline CSRP3 & 600824 & $11 p 15.1$ & Cysteine and glycine-rich protein 3-cardiac LIM protein & $\begin{array}{l}\text { DCM } \\
\text { HCM }\end{array}$ \\
\hline DES & 125660 & $2 q 35$ & Desmin & $\begin{array}{l}\text { MFM } \\
\text { DCM }\end{array}$ \\
\hline$D M D$ & 300377 & Xp21.2 & Dystrophin & $\begin{array}{l}\text { DMD } \\
\text { BMD } \\
\text { DCM }\end{array}$ \\
\hline DSC2 & 125645 & 18q12.1 & Desmocollin 2 & ARVC \\
\hline DSG2 & 125671 & $18 q 12.1$ & Desmoglein 2 & $\begin{array}{l}\text { ARVC } \\
\text { DCM }\end{array}$ \\
\hline$D S P$ & 125647 & $6 \mathrm{p} 24$ & Desmoplakin & $\begin{array}{l}\text { ARVC } \\
\text { DCM }\end{array}$ \\
\hline DTNA & 601239 & 18q12.1 & Dystrobrevin, alpha & LVNC \\
\hline$E M D$ & 300384 & Xq28 & Emerin & $\begin{array}{l}\text { EDMD } \\
\text { DCM }\end{array}$ \\
\hline EYA4 & 603550 & 6q23-q24 & Eyes absent homolog 4 & $\begin{array}{l}\text { DFNA } \\
\text { DCM }\end{array}$ \\
\hline FKTN & 607440 & 9q31 & Fukutin & $\begin{array}{l}\text { FCMD } \\
\text { DCM }\end{array}$ \\
\hline HSPB7 & 610692 & lp36.13 & Heat shock $27 \mathrm{kDa}$ protein family, member 7 & DCM \\
\hline$J U P$ & 173325 & $17 q 21$ & Junctional plakoglobin & $\begin{array}{l}\text { ARVC } \\
\text { ND }\end{array}$ \\
\hline LAMP2 & 309060 & Xq24 & Lysosomal-associated membrane protein 2 & $\begin{array}{c}\text { DD } \\
\text { HCM } \\
\text { DCM }\end{array}$ \\
\hline$\angle D B 3$ & 605906 & $10 q 22-q 23$ & LIM domain binding 3 & $\begin{array}{c}\mathrm{DCM} \pm \mathrm{LVNC} \\
\mathrm{MFM}\end{array}$ \\
\hline LMNA & 150330 & $1 \mathrm{q} 21.2$ & Lamin A/C & $\begin{array}{c}\text { DCM } \pm \text { CD } \\
\text { CMT2B1 } \\
\text { EDMD } \\
\text { HGPS } \\
\text { LGMD } \\
\text { MADA } \\
\text { FPLD2 }\end{array}$ \\
\hline MYBPC3 & 600958 & 11p11.2 & Myosin binding protein $\mathrm{C}$, cardiac & $\begin{array}{l}\text { DCM } \\
\text { HCM }\end{array}$ \\
\hline MYH6 & 160710 & $14 q 12$ & Myosin, heavy chain 6 , cardiac muscle, alpha & $\begin{array}{l}\text { DCM } \\
\text { HCM }\end{array}$ \\
\hline MYH7 & 160760 & $14 q 12$ & Myosin, heavy chain 7 , cardiac muscle, beta & $\begin{array}{l}\text { DCM } \\
\text { HCM } \\
\text { RCM }\end{array}$ \\
\hline MYL2 & 160781 & $12 q 23-q 24$ & Myosin, light chain 2, regulatory, cardiac, slow & $\mathrm{HCM}$ \\
\hline MYL3 & 160790 & 3p21.3-21.2 & Myosin, light chain 3, alkali; ventricular, skeletal, slow & HCM \\
\hline MYPN & 608517 & 10q21.1 & Myopalladin & DCM \\
\hline NEBL & 605491 & 10p13-p12 & Nebulette & DCM \\
\hline NEXN & 613121 & $1 p 31.1$ & Nexilin & $\begin{array}{l}\text { DCM } \\
\text { HCM }\end{array}$ \\
\hline PKP2 & 602861 & 12p11 & Plakophilin 2 & ARVC \\
\hline PKP4 & 604276 & $2 q 23-q 31$ & Plakophilin 4 & ARVC \\
\hline
\end{tabular}


significant genetic and allelic heterogeneity and variable expressivity. ${ }^{19}$ However, contrary to DCM, genetic testing in HCM using the current technology has a high detection rate because mutations in four sarcomeric genes, $\beta$-myosin heavy chain (MYH7), myosin binding protein-C (MYBPC3), cardiac troponin $\mathrm{T}$ (TNNT2), and cardiac troponin I (TNNI3), cause approximately $80 \%$ of all familial HCM cases. Mutations can also be identified in about $40 \%$ of sporadic and idiopathic cases of HCM. ${ }^{11}$ Although genotype-phenotype correlation is imperfect, it has been reported that mutations in $M Y H 7$ are associated with early onset disease, and in some cases, a more severe phenotype, while $M Y B P C 3$ mutations have been identified in subjects with later onset presentation, and TNNT2 mutations are associated with a high incidence of sudden cardiac death. ${ }^{20-21}$

HCM is a monogenic disorder in most cases, although double heterozygote mutations have been described and appear to be associated with earlier-onset and a more dramatic phenotype. ${ }^{11}$

Despite the high detection rate of clinical genetic testing in HCM, the lack of good genotype-phenotype correlation has lessened the clinical utility of genetic screening in affected patients. However, clinical genetic testing is quite useful for the screening of clinically affected and unaffected family members due to the lack of symptoms in most people with HCM and the relatively high risk of sudden death. Genetic counseling by trained professionals is highly recommended for accurate familial risk assessment and to reduce the potential psychological impact of the genetic testing.

\section{Arrhythmogenic right ventricular cardiomyopathy}

ARVC is a myocardial disease morphologically characterized by fibrosis with or without fatty infiltration and dilation of the right ventricle, along with thinning of the right ventricular wall, arrhythmias and sudden cardiac death. ${ }^{22}$ However, left ventricular involvement is being more commonly identified as well typically of the posterior lateral wall, in an agedependent fashion. ${ }^{22}$

ARVC is often diagnosed secondary to arrhythmias, palpitations, syncope, and aborted sudden death. ARVC can manifest in the early teenage years with the majority of patients being in their late teenage years or young adulthood, especially in athletes. ${ }^{23}$ ARVC is predominantly genetically determined and often inherited within families, mostly as an autosomal dominant trait with reduced penetrance and variable expression. ${ }^{24}$ However, autosomal recessive inheritance has been observed in individuals affected by Naxos disease presenting with ARVC, diffuse non-epi-
Table 1. Continued from previous page.

\begin{tabular}{|c|c|c|c|c|}
\hline Gene & OMIIM* & Locus & Gene name $^{\circ}$ & $\begin{array}{l}\text { Associated } \\
\text { phenotypes }^{\circ}\end{array}$ \\
\hline$P L N$ & 172405 & $6 q 22.1$ & phospholamban & DCM \\
\hline PRKAG2 & 602743 & 7q36.1 Prote & in kinase, AMP-activated, gamma 2 non-catalytic subunit & $\begin{array}{l}\mathrm{HCM} \pm \mathrm{WPW} \\
\mathrm{FCNCG}\end{array}$ \\
\hline PSEN1 & 104311 & $14 q 24.3$ & Presenilin 1 & $\begin{array}{c}\text { AD } \\
\text { DCM } \\
\text { FAI }\end{array}$ \\
\hline PSEN2 & 600759 & 1q31-q42 & Presenilin 2 & $\begin{array}{l}\text { AD-4 } \\
\text { DCM }\end{array}$ \\
\hline RBM20 & 613171 & 10q25.2 & RNA binding motif protein 20 & DCM \\
\hline RYR2 & 180902 & lq43 & Ryanodine receptor 2 & $\begin{array}{l}\text { ARVC } \\
\text { CPVT }\end{array}$ \\
\hline SCN5A & 600163 & 3p21 & odium channel, voltage-gated, type $\mathrm{V}$, alpha subunit & $\begin{array}{c}\text { BRS } \\
\text { LQTS } \\
\text { DCM } \pm \text { CD } \\
\text { SGCB }\end{array}$ \\
\hline$S G C D$ & 601411 & $5 q 33-q 34$ & Sarcoglycan, delta & $\begin{array}{l}\text { LGMD } \\
\text { DCM }\end{array}$ \\
\hline$T A Z$ & 300394 & $\mathrm{Xq} 28$ & Tafazzin & $\begin{array}{c}\text { BTS } \\
\text { LVNC } \\
\text { DCM }\end{array}$ \\
\hline TCAP & 604488 & 17q12 & Titin-cap-telethonin & $\begin{array}{c}\text { LGMD } \\
\text { HCM } \\
\text { DCM }\end{array}$ \\
\hline TGFB3 & 190230 & $14 q 24$ & Transforming growth factor, beta 3 & ARVC \\
\hline TMEM43 & 612048 & $3 p 25.1$ & Transmembrane protein 43 & ARVC \\
\hline TMPO & 188380 & $12 \mathrm{q} 22$ & Thymopoietin & DCM \\
\hline TNNCl & 191040 & $3 \mathrm{p} 21.1$ & Troponin C type 1 & $\begin{array}{l}\text { DCM } \\
\text { HCM }\end{array}$ \\
\hline TNNI3 & 191044 & 19q 13.4 & Troponin I type 3 & $\begin{array}{l}\text { DCM } \\
\text { HCM } \\
\text { RCM }\end{array}$ \\
\hline TNNT2 & 191045 & $1 q 32$ & Troponin T type 2 & $\begin{array}{l}\text { DCM } \\
\text { HCM } \\
\text { RCM }\end{array}$ \\
\hline TPM1 & 191010 & $15 q 22.1$ & Tropomyosin 1 & $\begin{array}{l}\text { DCM } \\
\text { HCM }\end{array}$ \\
\hline TTN & 188840 & $2 q 31$ & Titin & $\begin{array}{c}\text { TMD } \\
\text { LGMD } \\
\text { EOMFC } \\
\text { DCM } \\
\text { HCM } \\
\text { HMERF }\end{array}$ \\
\hline$V C L$ & 193065 & 10q22.1-q23 & Vinculin & $\begin{array}{l}\text { DCM } \\
\text { HCM }\end{array}$ \\
\hline
\end{tabular}

AD, Alzheimer disease; ARVC, arrhythmogenic right ventricular cardiomyopathy; BMD, Becker muscular dystrophy; BRS, Brugada syndrome; CD conduction defects; CMT2B1, Charcot-Marie-tooth disease type 2B1; CPVT, catecholaminergic polymorphic ventricular tachycardia; DCM, dilated cardiomyopathy; DD, Danon disease; DFNA, autosomal dominant late-onset progressive nonsyndromic deafness; DMD, Duchenne muscular dystrophy; EDMD, Emery-Dreifuss muscular dystrophy; EOMFC, early-onset myopathy with fatal cardiomyopathy; FAI, familial acne inverse; FCMD, Fukuyama congenital muscular dystrophy; FCNCG, fatal congenital nonlysosomal cardiac glycogenosis; FPLD2, familial partial lipodystrophy of the Dunnigan type; HCM, hypertrophic cardiomyopathy; HGPS, Hutchinson-Gilford progeria syndrome; HMERF, myopathy with early respiratory muscle involvement; LGMD, Limb-Girdle muscular dystrophy; LQTS, long QT syndrome; LVNC, left ventricular non-compaction; ND naxos disease; MADA, mandibuloacral dysplasia type A with partial lipodystrophy; MFM, myofibrillar myopathy; RCM, restrictive cardiomyopathy; TMD, tibial muscular dystrophy, tardive; WPW, Wolff-Parkinson-White syndrome. The symbol \pm indicates that a phenotype can be associated or not with other clinical presentations. *OMIM, Online Mendelian Inheritance in Man. http:/www.ncbi.nlm.nih.gov/omim Accessed: May 2011; ${ }^{\circ} \mathrm{Genetic} \mathrm{Home} \mathrm{References.} \mathrm{http://ghr.nlm.nih.gov/} \mathrm{Accessed:} \mathrm{May} 2011$. 
dermolytic palmoplantar keratoderma, and woolly hair, as well as in Carvajal syndrome, characterized by striate palmoplantar keratoderma, woolly hair, and LV cardiomyopathy. ${ }^{25}$ ARVC has an estimated prevalence of 1/20005000 , although regional differences may significantly vary this estimate worldwide..$^{25-26}$

Diagnostic assessment for ARVC includes cardiac MRI (CMR), echocardiography, and electrocardiography. The CMR classically demonstrates a dilated RV with RV wall thinning and fatty replacement of the myocardium, systolic dysfunction and the RV outflow tract may be aneurysmal. Gadolinium late enhancement typically identifies areas of fibrosis. The LV may also be affected with a DCM appearance. The echocardiogram may demonstrate a dilated RV or LV with systolic dysfunction and the electrocardiogram demonstrates $\mathrm{T}$ wave inversion in leads $V_{1}$ through $V_{3}$, QRS duration $110 \mathrm{msec}$ in $V_{1}$ through $V_{3}$, an epsilon wave (electric potentials after the end of the QRS complex), an ST-elevation pattern similar to that seen in Brugada syndrome may be noted, and a prolonged $S$-wave upstroke in $V_{1}$ through $\mathrm{V}_{3}$ measuring $55 \mathrm{msec}$. Right ventricular biopsy may be definitive when typical fibrofatty replacement of the myocardium and inflammation is visible; this is more obvious, in advanced disease. Commonly, only fibrosis and inflammatory infiltrates are seen, particularly in earlier disease presentations. Since the primary area of fibrofatty replacement is in the triangle of dysplasia in the apical and infundibular regions of the RV, making biopsy diagnosis more challenging. Autopsy or explant after transplantation will commonly demonstrate classic histopathologic diagnostic criteria. ${ }^{26-27}$

Currently, 13 loci and mutations in 9 genes have been identified in ARVC individuals (Table 1). Most of the genes causing ARVC encode proteins of the cardiac desmosome such as plakoglobin (PG), desmoplakin (DSP), plakophilin-2 (PKP2), plakophillin-4 (PKP4), desmocollin-2 (DSC2) and desmoglein-2 (DSG2), resulting in defective cell-to-cell adhesion and altered nuclear signaling, leading to diminished desmosomal protein localization and dramatic reduction in immunoreactive signal for the GJAl-encoded gap junction protein connexin-43 (Cx43) at the intercalated disks. ${ }^{25-28,29}$

Connexin- 43 is a highly phosphorylated protein, whose phosphorylation pattern plays important roles in the regulation of protein turnover, trafficking, intercalated disk assembly, internalization, degradation, and channel gating properties. In the failing $\mathrm{RV}$, altered phosphorylation of $\mathrm{Cx} 43$ leads to a non-phosphorylated protein, causing a weaker immunoreactive signal to be observed in the $\mathrm{RV}$ myocardium of patients and animal models with ARVC..$^{28,29}$ Recently, Gehmlich and col- leagues suggested that the cytoplasmic portion of a highly phosphorylated $\mathrm{Cx} 43$ protein binds the DSC2a isoform, connecting the gap junction to the desmosome. ${ }^{28}$ They identified a novel variant in DSC2 as well as a DSG2 variant in an individual with a family history of sudden death, mild ECG abnormalities in herself and her daughter, and immunohistochemistry demonstrated severe depression of the PG signal at the intercalated disk, whereas Western blot showed minimal reduction of DSG2 and DSC2 expression levels and mild reduction of $\mathrm{Cx} 43$. Electrophoretic mobility of Cx43 was abnormal and consistent with differential phosphorylation, suggesting a lower proportion of the highly phosphorylated protein. All other desmosomal proteins were normal. Therefore, the authors suggested that the DSC2a isoform provides a critical link between the desmosome and gap junction and that disruption leads to the clinical features of disease upon physiologic trigger. They also suggested that the combination of PG loss and Cx43 disturbance is an early indicator of developing clinical disease, with the risk of arrhythmias (and therefore sudden death) lurking for future clinical presentation. An extension of this suggestion leads to the possibility that mutations in desmosomal proteins could possibly alter $\mathrm{Cx} 43$ phosphorylation and weaken the linkage between the gap junction and desmosome, causing loss of electrical coupling between cardiac myocytes, and leading to myocyte cell death, fibrofatty replacement and arrhythmias. We suggested that, if correct, these findings could potentially be used for early genotype-phenotype biomarker disease diagnosis, risk stratification and outcome prediction, and possibly preventive therapy, but that significant caution should be used before assuming this to be correct. ${ }^{22}$

In addition to abnormalities in desmosomal genes, mutations in the TGF $33, R Y R 2$, and TMEM43 genes have been also associated with ARVC and are thought to cause ARVC via secondary disruption of the desmosome. ${ }^{27}$ The TGF $\beta 3$-encoded transforming growth factor $\beta 3$ is a cytokine, which stimulates fibrosis and modulates cell adhesion, while the RYR2encoded human ryanodine receptor 2 induces the release of calcium from the myocardial sarcoplasmic reticulum. TMEM43, which encodes transmembrane protein 43 , is a response element for the peroxisome proliferator-activated receptor gamma (PPAR $\gamma$ ) gamma, an adipogenic transcription factor, which may explain the fibrofatty replacement of the myocardium. $^{27}$

Although most ARVC cases follow an autosomal dominant pattern, autosomal recessive pattern was recognized with homozygous mutations in the plakoglobin-encoding gene causing Naxos disease, and homozygous mutations in DSP in Carvajal syndrome..$^{26,27}$
However, because of the reduced penetrance and variable expressivity, which characterizes ARVC, single mutations in individual genes may not be sufficient to cause the development of the disease.22,25 Compound heterozygous mutations or double heterozygous, digenic mutations in desmosomal genes may be required for disease development and clinical manifestation. ${ }^{24,27}$ In fact, although frameshift mutations are regarded as deleterious changes according to the current recommendations, ${ }^{30}$ many PKP2 mutations demonstrate low penetrance and an additional mutation in another ARVC gene is necessary to develop the disease. Therefore, despite much literature suggesting that PKP2 mutations cause approximately $25 \%$ of ARVC cases, this is not the primary cause of the disease in a high percentage of subjects. This complex genetic behavior makes clinical genetic testing challenging to interpret. Therefore, despite the apparent autosomal dominant mode of inheritance, clinical genetic testing should be comprehensive and multimodal for all the known ARVC genes and genetic-based diagnosis should be conservatively and thoughtfully considered.

\section{Left ventricular noncompaction}

Left ventricular non compaction (LVNC) is characterized by a trabeculated left ventricular myocardium associated with deep inter-trabecular recesses in the left ventricular wall, typically most evident in the apical region of the $\mathrm{LV}$ but also notable in the LV free wall and, occasionally the septum in some patients. The RV, which normally is trabeculated, may be hypertrabeculated as well in some cases, resulting in biventricular noncompaction. The diagnosis is typically made by echocardiography where apical trabeculations are noted with or without trabeculations in the free wall, septum or RV. Some published criteria use a ratio of compact to noncompact layer of the LV myocardium $>2$ but this measurement is dependent on the area sampled and measured. In addition, cardiac MRI may demonstrate the trabeculations and inter-trabecular recesses well. Due to the trabeculations and inter-trabecular recesses there is a theoretical increase in the susceptibility to form clots in the LV with high risk of thromboembolism and death, but this usually occurs only in subjects with a dilated and poorly functioning $\mathrm{LV} .{ }^{31} \mathrm{LVNC}$ can be observed with or without congenital heart diseases (CHD) such as ventricular (VSD) and atrial septum defects (ASD), pulmonic stenosis (PS), Ebstein's anomaly, and hypoplastic left heart syndrome (HLHS), amongst others. When not associated with $\mathrm{CHD}$, the associated phenotype may include a DCM-like, HCM-like, combined HCM/DCM-like picture, a restrictive cardiomyopathy (RCM)-like picture, or simply LV trabeculations with normal LV size, thickness and function. ${ }^{32,33}$ The latter phenotype appears 
to be benign unless associated with arrhythmias while the other forms tend to follow a similar course to the associated phenotype. The only exception is the undulating phenotype, a change from one appearance to another, which most commonly occurs in those with the combined HCM/DCM-like picture. LVNC can also present associated with syndromes such as Barth, ${ }^{31}$ Marfan, ${ }^{34}$ Sotos,${ }^{35}$ trisomy 13, 36 and monosomy of chromosome band 1p $36^{37,38}$ as well as Duchenne and Becker muscular dystrophy. LVNC is believed to occur due to an arrest in the ventricular development during embryogenesis, in which the initially trabeculated myocardium provides enough surface for the blood to infiltrate and bring oxygen and nutrients to myocardial cells. ${ }^{31}$ Subsequently, with a higher blood demand, the coronary artery system develops and the myocardium undergoes remodeling associated with compaction of the hypertrabeculated myocardium. The disruption of this process is believed to cause the ventricular noncompaction. ${ }^{31}$

LVNC was considered a rare disease in the past due to limited awareness and knowledge of the disease and limitations of echocardiography. ${ }^{32}$ However, the American Heart Association recently classified LVNC as a separate clinical entity. ${ }^{3}$ The actual incidence and prevalence of LVNC remains unclear, but the reported incidence of $0.05 \%$ in isolated LVNC in adult subjects appears to be underestimated, and is probably far lower than the true incidence and prevalence in children.,31 In fact, LVNC is now assumed to account for approximately $10 \%$ of all cardiomyopathies. Currently, mutations in many genes have been identified in LVNC (Table 1), but the genes more commonly associated with LVNC in children are sarcomere-encoding genes, TAZ, DTNA and $L D B 3^{31,32,39}$ The TAZ gene, located on chromosome Xq28, encodes the tafazzin protein believed to function as phospholipidslysophospholipid transacylase involved in cardiolipin metabolism and part of the inner mitochondrial membrane. ${ }^{31}$ The TAZ gene was initially identified in patients with Barth syndrome, an X-linked disease characterized by cardiomyopathy, skeletal myopathy, cyclic neutropenia, abnormal cholesterol metabolism, lactic acidosis, 3-methylglutaconic aciduria, and cardiolipin abnormalities. ${ }^{33}$ More recently, $T A Z$ mutations have been identified in subjects presenting with isolated LVNC, and endocardial fibroelastosis and isolated LVNC. ${ }^{32}$

$\alpha$-Dystrobrevin is a cytoskeletal protein encoded by the DTNA gene and is a component of the dystrophin associated glycoprotein complex, which provides structural stability during muscle contraction and relaxation. The LIMdomain binding protein 3 (LDB3), also known as ZASP (Z-band alternatively spliced PDZmotif protein), is a component of the sarcomeric Z-line and is encoded by the $L D B 3$ gene and characterized by one PDZ domain at its $\mathrm{N}$ terminus and up to three LIM domains at its Cterminus.40 Mutations in these genes cause LVNC with or without CHD. ${ }^{39}$

\section{Restrictive cardiomyopathy}

Restrictive cardiomyopathy (RCM) is uncommon, accounting for up to $5 \%$ of cardiomyopathies in children ${ }^{41}$ Restrictive cardiomyopathies are characterized by dilated atria with normal ventricular size, thickness and systolic function in the face of diastolic ventricular dysfunction with elevated left (and at times right) ventricular end-diastolic pressures. ${ }^{42-43}$ In the 2006 classification consensus statement by the AHA, primary restrictive cardiomyopathy was defined as a rare form of heart disease characterized by normal or decreased volume of both ventricles associated with biatrial enlargement, normal left ventricular wall thickness and atrioventricular valves, impaired ventricular filling with restrictive physiology, and normal (or near normal) systolic function. ${ }^{3}$

The most common presenting signs and symptoms in children with RCM include dyspnea that is frequently exacerbated by an intercurrent respiratory illness or asthma, fatigue, exercise intolerance, syncope, and sudden death. ${ }^{44-45}$ The electrocardiogram is abnormal in approximately $98 \%$ of patients. ${ }^{46-48}$ The most common abnormalities are right and/or left atrial enlargement; however ST segment depression and ST-T wave abnormalities are frequently present. Right and/or left ventricular hypertrophy can also be seen as well as conduction abnormalities. Holter and event monitors are useful to evaluate for rhythm disturbances, conduction abnormalities and evidence of ischemia based on ST segment analysis. ${ }^{46-48}$ Arrhythmias have been reported in approximately $15 \%$ of pediatric patients and include atrial flutter, high grade second and third degree atrioventricular block, atrial fibrillation, atrial tachycardias, Wolff-ParkinsonWhite syndrome with supraventricular tachycardia and ventricular tachycardia and torsades. Symptomatic sinus bradycardia requiring pacing has also been reported. ${ }^{47-48}$ The most striking finding on echocardiography is massive atrial dilatation in the absence of atrioventricular valve regurgitation. ${ }^{49}$ In children, findings consistent with restrictive filling and increased left ventricular end diastolic pressure are noted. ${ }^{50}$ Systolic function is typically preserved although some degree of systolic dysfunction has been seen in some patients at presentation and deterioration of systolic dysfunction over time has also been reported in children. $^{51}$ Ventricular hypertrophy is not prominent, but some degree of concentric increase in septal and left ventricular posterior wall thickness is seen in a significant proportion of cases otherwise fulfilling all the other criteria for RCM. Cardiac catheterization may demonstrate elevated LV or RV end diastolic pressures, pulmonary hypertension with elevation in pulmonary artery pressure is frequently present at the time of initial catheterization and markedly elevated pulmonary vascular resistance can occur within 1 to 4 years of diagnosis. ${ }^{49}$ Endomyocardial biopsy reveals myofiber hypertrophy and mild to moderate interstitial fibrosis.

In children in the U.S. and Australia, RCM accounts for $2.5-5 \%$ of the diagnosed cardiomyopathies, with the majority having no specific cause identified. 41,52 In Australia, RCM accounted for $2.5 \%$ of the cardiomyopathies diagnosed in children $<10$ years of age ${ }^{53}$ while the U.S report from the Pediatric Cardiomyopathy Registry (PCMR) investigators reported that RCM accounted for $3 \%$ of the cardiomyopathies in children $<18$ years of age. ${ }^{22}$ The estimated annual incidence in the U.S. and Australia is $0.04 / 100,000$ and $0.03 / 100,000$ children, respectively. ${ }^{52,53}$ Multiple causes of RCM have been described in adults and children.

Some cases are inherited and most commonly have autosomal dominant transmission. Causative mutations have been reported in sarcomere-encoding genes such as troponin I, troponin T, $\beta$-myosin heavy chain, and actin..$^{54}$ Another complex subgroup of patients have been identified with RCM associated with atrioventricular block and skeletal myopathy, and these are usually caused by mutations in desmin or lamin A/C. ${ }^{54}$

The prognosis in children with RCM is poor. ${ }^{41,55}$ Half of the children die or undergo transplant within 3 years of diagnosis. ${ }^{41,55}$ Sudden cardiac death has been reported to be a common mode of death in children with RCM. ${ }^{46}$ Patients who appear to be at greater risk for sudden death include those who present with signs and symptoms of ischemia, such as syncope and chest pain. However, heart failure related deaths are the most common.

\section{Metabolic cardiomyopathies}

Although the major focus of this review is the description of primary cardiomyopathies, which present usually in isolation affecting only the cardiac muscle, defects in the metabolism usually appear early in life and may lead to complex clinical phenotype in which the function of multiple tissues including the myocardium results severely hampered. Metabolic cardiomyopathies can be recognized as early as the perinatal period or infancy, and among the best known there are approximately 50 different forms of lysosomal accumulation of substrates, which results to be toxic for the cell, namely the lysosomal storage diseases (LSDs) ${ }^{56}$ Among the various LSD here we will mention Pompe disease, Fabry disease and 
Gaucher disease. According to their age of onset, variable clinical presentation and severity, they are also divided in various sub-types. Pompe disease is an autosomal recessive disorder caused by mutations in the $G A A$ gene mapping to chromosome 17q25.2-q25.3 and encoding the enzyme acid alpha-glucosidase, which is essential for the degradation of glycogen to glucose in the lysosomes. Defects in this process lead to glycogen storage disease type II with the toxic accumulation in various organs such as striated muscles, impairing their normal function..$^{5}$ Pompe disease has an estimated prevalence of 1: 40,000 and may present as early as in infancy with progressive left ventricular hypertrophy, but late-onset forms such as in late adolescents and young adults may not present with heart failure. However, subjects with Pompe disease present a significant risk of sudden cardiac death due to the association of hypertrophic cardiomyopathy along with Wolff-Parkinson-White syndrome, a form of atrioventricular reentrant tachycardia or ventricular pre-excitation. ${ }^{56}$

Fabry disease is an X-linked form of LSD caused by impaired activity of the enzyme $\alpha$ galactosidase ( $\alpha$-Gal A) due to mutations in the $G L A$ gene, mapping to chromosome Xq22. ${ }^{56}$ Subejcts with Fabry disease accumulate glycosphingolipid ceramide trihexoside (GL-3), which is deposited into lysosomes in most cell types in the body.

The prevalence of Fabry disease is approximately $1: 40,000$, although the prevalence may be much higher in subsets of patients. ${ }^{56}$ Fabry disease classically occurs in hemizygote males in early childhood or adolescence with periodic crises of severe pain in the extremities (acroparesthesias), vascular cutaneous lesions (angiokeratomas), kidney failure, corneal and lenticular opacities, proteinuria and heart failure or death. ${ }^{56}$

However, Fabry disease may also present with HCM as the prominent symptom although restrictive cardiomyopathy has also been observed.

Gaucher disease (GD) is an autosomal recessive LSD caused by mutations in the $G B A$ gene (1q21) leading to deficient activity of the encoded acid- $\beta$-glucosidase. Gaucer disease has a prevalence of approximately in 1: 50,000 to 100,000 people in the general population and can present as a perinatal lethal disorder, although the variable clinical presentation include some mild or sub-clinical forms. ${ }^{56}$

However, GD Type 1 is the most common form of the disorder, occurring more frequently in individuals with Ashkenazi Jewish descent compared to the other genetic backgrounds, affecting 1: 500 to 1000 Ashkenazi Jews. ${ }^{56}$

Another form of LSDs recently classified in the subgroup of autophagic vacuolar myopathies (AVMs) and that can present with
HCM as its predominant feature is represented by Danon disease and it is caused by mutations in the LAMP2 gene, which encodes the lyosome-associated protein-2 and maps to Xq24. ${ }^{57-58}$ Affected hemizygote males usually present with severe cardiac hypertrophy in early childhood and adolescence, while most female carriers develop dilated cardiomyopathy (DCM) rather than HCM during adulthood (as late as their 40s). ${ }^{57-58}$ However, DCM has also been observed in males with Danon disease. ${ }^{58}$ Danon disease individuals suffer of various degree of intellectual disability and skeletal muscle impairment. Skeletal muscle biopsy usually reveals glycogen containing (PAS positive) cytoplasmic vacuoles. ${ }^{57-58}$

Because LSDs are caused by the deficiency in enzyme activity, this prompted the researchers to develop an enzyme replacement therapy (ERT) using recombinant enzyme, which appears to be effective when administered early in the natural history of the disease. Unfortunately, the enzymes do not pass the brain-blood barrier and they are not effective in ameliorating the neurological symptoms..$^{51,56}$

\section{Mitochondrial genome and nuclear genes with mitochondrial function and cardiomyopathies}

Mitochondria provide the major energy source for the myocardium, and abnormalities of the mitochondrial genome (mtDNA) and nuclear genes encoding proteins involved in mitochondrial respiratory chain function are seen in cardiomyopathies. The mitochondrial respiratory chain consists in five enzyme complexes (I-V) in the inner membrane of the mitochondria and the energy that is generated is used to produce ATP via oxidative phosphorylation. ${ }^{59,60}$ Defects in oxidative phosphorylation could originate from alterations in any of the five complexes of the respiratory chain, although the most frequently affected includecomplexes I (NADH-CoQ reductase) and IV (cytochrome-c oxidase). ${ }^{60}$ Mitochondrial diseases usually manifest in early childhood, although different levels of heteroplasmy in various tissues may be associated with pleiotropic effects clinically and age-dependent expressivity. Children affected by mitochondrial diseases commonly present with cardiomyopathy (17\%) and cardiac involvement is generally associated with worse prognosis and increased mortality. ${ }^{59-61}$

Mitochondria occupy a significant portion (20-30\%) of cardiac cell volume, and their dynamic nature allow them to respond to the level of energy requirement by altering their unit number. ${ }^{59}$ In cardiomyocytes, mitochondria are not only functionally related to cardiac contraction and energy homeostasis, but they are also physically linked to the sarcoplasmic reticulum (SR) through the intermediate filament (IF) protein desmin, which provides the necessary ratio of mitochondria to IF for the optimal exchange of ions, lipids, and other metabolites. $^{61}$

Thus, it should not be surprising that altered integrity of the mitochondria could lead to functional and structural impairment of cardiac cells. In fact, the absence of desmin in mouse hearts leads to an abnormal accumulation of subsarcolemmal clusters of mitochondria, degeneration of the mitochondrial matrix, and proliferation associated with the development of dilated cardiomyopathy and heart failure. ${ }^{61}$

The mtDNA is approximately $16 \mathrm{~kb}$ in length and encodes 13 of the 69 proteins required for oxidative metabolism performed by Complex IV, 22 transfer RNA (tRNAs) and two ribosomal RNA (rRNA). Mitochondria are inherited from the mother and they can vary in number within the cells from various tissues (heteroplasmy), accounting for the pleiotropic effect. Recently, mutations leading to mitochondrial defects (mtDNA, nDNA), have been identified in subjects with isolated LVNC, ${ }^{62}$ as well as DCM and HCM, ${ }^{63}$ thus supporting the importance of mitochondrial genome screening in primary cardiomyopathies. In addition, complex mitochondrial diseases such as KearnsSayre syndrome, myoclonic epilepsy with ragged red muscle fibers (MERRF) ${ }^{33}$ syndrome and mitochondrial encephalopathy, lactic acidosis, and stroke-like episodes (MELAS) ${ }^{64}$ have also been associated with cardiomyopathies, suggesting that a multimodal approach including novel technologies for the screening of the nuclear DNA (nDNA) and the mtDNA should be employed in both research and clinical testing in individuals with cardiomyopathies, particularly babies. The currently available technologies such as whole genome or exome sequencing, MLPA, aCGH for nDNA and mtDNA and high density array combining single nucleotide polymorpshism (SNP) and copy number variation (CNV) probes using the currently available platforms could allow for rapid results with increasingly lower cost, improvements in turn-around times, and facilitating comprehensive investigation of the underlying genetic etiologies involved in cardiomyopathies.

\section{Final remarks}

In this review article we discussed the current knowledge about the genetics of cardiomyopathies in children. Despite over three decades of research and the discovery of a large number of causative genes, modest advances have been made about the management and therapies of cardiomyopathies in children as well as in adults. However, the advances in the genetics of heart diseases provided a formidable tool for counseling car- 
diomyopathy patients and their relatives to identify at-risk individuals and to evaluate the recurrent risk of the disease within the family. In addition, the use of more sophisticated ultrasound technology for in utero diagnosis, and newborn screening for postnatal ascertainment, have provided the clinicians with an unprecedented ability to obtain an early detection of the disease before symptoms appear and possibly, in the case of metabolic defects, employ prompt therapeutic intervention to halt or slow down the pathological process in perinatal and infant forms of cardiomyopathy. Much remains to be done, and an integrated approach using whole genome or exome sequencing, high density SNP arrays along with epigenetic platforms, may provide a better comprehension of the genetic mechanisms leading to the development of cardiomyopathies.

\section{References}

1. Heron M, Sutton PD, Xu J, et al. Annual summary of vital statistics: 2007. Pediatrics 2010;125:4-15.

2. Lipshultz SE, Sleeper LA, Towbin JA, et al. The incidence of pediatric cardiomyopathy in two regions of the United States. N Engl J Med 2003;348:1647-55.

3. Maron BJ, Towbin JA, Thiene G, et al. Contemporary definitions and classification of the cardiomyopathies: an American Heart Association Scientific Statement from the Council on Clinical Cardiology, Heart Failure and Transplantation Committee; Quality of Care and Outcomes Research and Functional Genomics and Translational Biology Interdisciplinary Working Groups; and Council on Epidemiology and Prevention. Circulation 2006;113:1807-16.

4. Elliott P, Andersson B, Arbustini E, et al. Classification of the cardiomyopathies: a position statement from the European Society Of Cardiology Working Group on Myocardial and Pericardial Diseases. Eur Heart J 2008;29:270-6.

5. Hsu DT, Canter CE. Dilated cardiomyopathy and heart failure in children. Heart Fail Clin 2010;6:415-32.

6. Towbin JA, Lowe AM, Colan SD, et al. Incidence, causes, and outcomes of dilated cardiomyopathy in children. JAMA 2006; 296:1867-76.

7. Ghosh N, Haddad H. Recent progress in the genetics of cardiomyopathy and its role in the clinical evaluation of patients with cardiomyopathy. Curr Opin Cardiol 2011; 26:155-64.

8. Mestroni L, Rocco C, Gregori D, et al. Familial dilated cardiomyopathy: evidence for genetic and phenotypic heterogeneity. Heart Muscle Disease Study Group. J Am Coll Cardiol 1999;34:181-90.

9. Towbin JA, Hejtmancik JF, Brink P, et al. Xlinked dilated cardiomyopathy. Molecular genetic evidence of linkage to the Duchenne muscular dystrophy (dystrophin) gene at the Xp21 locus. Circulation 1993;87:1854-65.

10. Towbin, JA. The role of cytoskeletal proteins in cardiomyopathies. Curr Opin Cell Biol 1998;10:131-9.

11. McBride KL, Garg V. Impact of Mendelian inheritance in cardiovascular disease. Ann N Y Acad Sci 2010;1214:122-37.

12. Hermans MC, Pinto YM, Merkies IS, et al. Hereditary muscular dystrophies and the heart. Neuromuscul Disord 2010;20:47992.

13. Finsterer J, Ramaciotti C, Wang CH, et al. Cardiac findings in congenital muscular dystrophies. Pediatrics 2010;126:538-45.

14. Cheng J, Morales A, Siegfried JD, et al. SCN5A rare variants in familial dilated cardiomyopathy decrease peak sodium current depending on the common polymorphism H558R and common splice variant Q1077del. Clin Transl Sci 2010;3:28794.

15. Li Z, Ai T, Samani K, et al. A ZASP missense mutation, S196L, leads to cytoskeletal and electrical abnormalities in a mouse model of cardiomyopathy. Circ Arrhythm Electrophysiol 2010;3:646-56.

16. Norton N, Li D, Rieder MJ, et al. Genomewide studies of copy number variation and exome sequencing identify rare variants in BAG3 as a cause of dilated cardiomyopathy. Am J Hum Genet 2011;88:273-82.

17. Villard E, Perret C, Gary F, et al. A genomewide association study identifies two loci associated with heart failure due to dilated cardiomyopathy. Eur Heart J 2011;32:106576 .

18. Kaski JP, Syrris P, Esteban MT, et al. Prevalence of sarcomere protein gene mutations in preadolescent children with hypertrophic cardiomyopathy. Circ Cardiovasc Genet 2009;2:436-41.

19. Schwartz ML, Cox GF, Lin AE, et al. Clinical approach to genetic cardiomyopathy in children. Circulation 1996;94:2021-38.

20. Morita H, Rehm HL, Menesses A, et al. Shared genetic causes of cardiac hypertrophy in children and adults. N Engl J Med 2008;358:1899-908.

21. Frisso G, Limongelli G, Pacileo G, et al. A child cohort study from southern Italy enlarges the genetic spectrum of hypertrophic cardiomyopathy. Clin Genet 2009;76:91-101.

22. Towbin JA. Desmosomal gene variants in patients with "possible ARVC". Heart Rhythm 2011;8:719-20.
23. Sequeira IB, Kirsh JA, Hamilton RM, et al. Utility of exercise testing in children and teenagers with arrhythmogenic right ventricular cardiomyopathy. Am J Cardiol 2009;104:411-3.

24. Xu T, Yang Z, Vatta M, et al. Compound and digenic heterozygosity contributes to arrhythmogenic right ventricular cardiomyopathy. J Am Coll Cardiol 2010;55: 587-97.

25. Vatta M, Marcus FI, Towbin JA. Arrhythmogenic right ventricular cardiomyopathy: a "final common pathway" that defines clinical phenotype. Eur Heart J 2007;28:529-30.

26. Corrado D, Thiene G. Arrhythmogenic right ventricular cardiomyopathy/dysplasia: clinical impact of molecular genetic studies. Circulation 2006;113:1634-7.

27. Azaouagh A, Churzidse S, Konorza T, Erbel R. Arrhythmogenic right ventricular cardiomyopathy/dysplasia: a review and update. Clin Res Cardiol 2011;100:383-94.

28. Gehmlich K, Lambiase PD, Asimaki A, et al. A novel desmocollin-2 mutation reveals insights into the molecular link between desmosomes and gap junctions. Heart Rhythm 2011;8:711-8.

29. Sasano $\mathrm{C}$, Honjo $\mathrm{H}$, Takagishi $\mathrm{Y}$, et al. Internalization and dephosphorylation of connexin43 in hypertrophied right ventricles of rats with pulmonary hypertension. Circ J 2007;71:382-9.

30. Richards CS, Bale S, Bellissimo DB, et al. ACMG recommendations for standards for interpretation and reporting of sequence variations: Revisions 2007. Genet Med 2008;10:294-300.

31. Weisz SH, Limongelli G, Pacileo G, et al. Left ventricular non compaction in children. Congenit Heart Dis 2010;5:384-97.

32. Ritter M, Oechslin E, Sütsch G, et al. Isolated noncompaction of the myocardium in adults. Mayo Clin Proc 1997;72:2631.

33. Towbin JA. Left ventricular noncompaction: a new form of heart failure. Heart Fail Clin 2010;6:453-69.

34. Kwiatkowski D, Hagenbuch S, Meyer R. A teenager with Marfan syndrome and left ventricular noncompaction. Pediatr Cardiol 2010;31:132-5.

35. Martinez HR, Belmont JW, Craigen WJ, et al. Left ventricular noncompaction in Sotos syndrome. Am J Med Genet A 2011;155:1115-8.

36. McMahon CJ, Chang AC, Pignatelli RH, et al. Left ventricular noncompaction cardiomyopathy in association with trisomy 13. Pediatr Cardiol 2005;26:477-9.

37. Dod HS, Bhardwaj R, Hummel M, et al. Left ventricular noncompaction: a rare disorder in adults and its association with 1p36 chromosomal anomaly. Am J Med Genet A 
2010;152A:191-5.

38. Gajecka M, Saitta SC, Gentles AJ, et al. Recurrent interstitial 1p36 deletions: Evidence for germline mosaicism and complex rearrangement breakpoints. Am J Med Genet A 2010;152A:3074-83.

39. Vatta M, Mohapatra B, Jimenez S, et al. Mutations in Cypher/ZASP in patients with dilated cardiomyopathy and left ventricular non-compaction. J Am Coll Cardiol 2003;42:2014-27.

40. Faulkner G, Pallavicini A, Formentin E, et al. ZASP: a new Z-band alternatively spliced PDZ-motif protein. J Cell Biol 1999;146:465-75.

41. Denfield SW, Webber SA. Restrictive cardiomyopathy in childhood. Heart Fail Clin 2010;6:445-52.

42. Lewis AB. Clinical profile and outcome of restrictive cardiomyopathy in children. Am Heart J 1992;123:1589-93.

43. Denfield SW, Rosenthal G, Gajarski RJ, et al. Restrictive cardiomyopathies in childhood: Etiologies and natural history. Tex Heart Inst J 1997;24:38-44.

44. Cetta F, O'Leary PW, Seward JB, Driscoll DJ. Idiopathic restrictive cardiomyopathy in childhood: diagnostic features and clinical course. Mayo Clin Proc 1995;70:63440.

45. Russo LM, Webber SA. Idiopathic restrictive cardiomyopathy in children. Heart 2005;91:1199-202.

46. Rivenes SM, Kearney DL, Smith EO, et al. Sudden death and cardiovascular collapse in children with restrictive cardiomyopathy. Circulation 2000;102:876-82.

47. Fitzpatrick AP, Shapiro LM, Rickards AF,
Poole-Wilson PA. Familial restrictive cardiomyopathy with atrioventricular block and skeletal myopathy. Br Heart J 1990; 63:114-8

48. Denfield SW. Sudden death in children with restrictive cardiomyopathy. Card Electrophysiol Rev 2002;6:163-7.

49. Nihoyannopoulos P, Dawson D. Restrictive cardiomyopathies. Eur J Echocardiogr 2009;10:iii23-33.

50. Chen SC, Balfour IC, Jureidini S. Clinical spectrum of restrictive cardiomyopathy in children. J Heart Lung Transplant 2001;20: 90-2.

51. Neudorf U, Bolte A, Lang D, et al. Diagnostic findings and outcome in children with primary restrictive cardiomyopathy. Cardiol Young 1996;6:44-7.

52. Nugent AW, Daubeney PE, Chondros P, et al. The epidemiology of childhood cardiomyopathy in Australia. N Engl $\mathrm{J}$ Med 2003;348:1639-46.

53. Sen-Chowdhry S, Syrris P, McKenna WJ. Genetics of restrictive cardiomyopathy. Heart Fail Clin 2010;6:179-86.

54. Weller RJ, Weintraub R, Addonizio LJ, et al. Outcome of idiopathic restrictive cardiomyopathy in children. Am J Cardiol 2002;90:501-6.

55. Chatterjee K. Diastolic ventricular failure: A clinician's approach. ACC Curr J Rev 1995;4:50-2.

56. Wang RY, Bodamer OA, Watson MS, et al. Lysosomal storage diseases: diagnostic confirmation and management of presymptomatic individuals. Genet Med 2011;13:457-84.

57. Yang Z, Vatta M. Danon disease as a cause of autophagic vacuolar myopathy. Congenit Heart Dis 2007;2:404-9.

58. Taylor MR, Ku L, Slavov D, et al. Danon disease presenting with dilated cardiomyopathy and a complex phenotype. J Hum Genet 2007;52:830-5.

59. Ren J, Pulakat L, Whaley-Connell A, Sowers JR. Mitochondrial biogenesis in the metabolic syndrome and cardiovascular disease. J Mol Med (Berl) 2010;88:9931001.

60. Holmgren D, Wåhlander H, Eriksson B0, et al. Cardiomyopathy in children with mitochondrial disease; clinical course and cardiological findings. Eur Heart J 2003; 24:280-8.

61. Capetanaki Y, Bloch RJ, Kouloumenta A, et al. Muscle intermediate filaments and their links to membranes and membranous organelles. Exp Cell Res 2007; 313: 2063-76.

62. Tang S, Batra A, Zhang Y, et al. Left ventricular noncompaction is associated with mutations in the mitochondrial genome. Mitochondrion 2010;10:350-7.

63. Sibbing D, Pfeufer A, Perisic T, et al. Mutations in the mitochondrial thioredoxin reductase gene TXNRD2 cause dilated cardiomyopathy. Eur Heart J 2011;32:112133.

64. Sproule DM, Kaufmann P. Mitochondrial encephalopathy, lactic acidosis, and strokelike episodes: basic concepts, clinical phenotype, and therapeutic management of MELAS syndrome. Ann N Y Acad Sci 2008;1142:133-58. 